\title{
Cierre transcatéter de fugas paravalvulares: resultados a corto y mediano plazo
}

\author{
Transcatheter closure of paravalvular leaks: short and medium-term outcomes
}

\author{
Piero Custodio-Sánchez ${ }^{*}$, José A. García-Montes ${ }^{1}$, Juan P. Sandoval', José A. Arias-Godínez², \\ Carlos Zabal-Cerdeira1', Eduardo A. Arias ${ }^{1}$, Alejandro Solórzano-Vásquez ${ }^{1}$, Hugo Zanella-Rodríguez², \\ Carlos Guerrero-Maya ${ }^{1}$ y Guillermo Aristizábal' \\ ${ }^{1}$ Departamento de Cardiología Intervencionista; ${ }^{2}$ Departamento de Ecocardiografía, Instituto Nacional de Cardiología Ignacio Chávez. Ciudad de
} México, México

\begin{abstract}
Resumen
Introducción: La fuga paravalvular es una complicación frecuente e importante posterior al reemplazo valvular quirúrgico que puede ocasionar insuficiencia cardiaca, anemia hemolítica y se relaciona con malos resultados clínicos. La reintervención quirúrgica ha sido el tratamiento habitual, pero se acompaña de alta morbimortalidad. El cierre transcatéter es una alternativa terapéutica. El objetivo del presente estudio es analizar la factibilidad y los resultados a corto y mediano plazo del cierre transcatéter de fugas paravalvulares con dispositivos oclusores. Métodos: Registro unicéntrico de una serie consecutiva de pacientes con fugas paravalvulares posquirúrgicas que fueron cerradas vía transcatéter con dispositivos oclusores, entre enero del 2006 y diciembre del 2016. Se analizaron los resultados de eficacia y seguridad durante el procedimiento y a seis meses. Resultados: Se trataron 21 fugas paravalvulares (15 mitrales, 5 aórticas y 1 tricuspídea) durante 20 procedimientos. El $91 \%$ de las fugas fue grave en la ecocardiografía inicial. El dispositivo utilizado con más frecuencia fue el Amplatzer Vascular Plug $11{ }^{\circledR}$ en 10 procedimientos (50\%). Se utilizó ecocardiografía transesofágica tridimensional en $70 \%$ de los casos. Se logró implantar el dispositivo con éxito en el $95 \%$ de los casos; se consiguió una reducción $\geq 1$ del grado de regurgitación en el $95 \%$ de las veces y se alcanzó el éxito clínico en el $79 \%$. A seis meses la supervivencia fue del 100\%; sin embargo, tres casos requirieron cirugía valvular (15\%). Conclusiones: El cierre transcatéter de fugas paravalvulares es un procedimiento factible, seguro y con tasas elevadas de éxito técnico, ecocardiográfico y clínico a corto y mediano plazo. Es una alternativa terapéutica adecuada, en particular en pacientes considerados de alto riesgo quirúrgico y múltiples comorbilidades.
\end{abstract}

Palabras clave: Fuga paravalvular. Cierre transcatéter. Resultados.

\section{Abstract}

Background: Paravalvular leak is a frequent and important complication after surgical valvular replacement that can cause heart failure, hemolytic anemia and is associated with poor clinical outcomes. Surgical reoperation has been the standard treatment, but it is associated with high morbidity and mortality. Transcatheter closure is a therapeutic alternative. The aim

Correspondencia:

*Piero Custodio-Sánchez

E-mail: custodiomed@ hotmail.com
Disponible en internet: 00-00-2020 Arch Cardiol Mex. 2020;90(2):122-129 www.archivoscardiologia.com 1405-9940/@ 2019 Instituto Nacional de Cardiología Ignacio Chávez. Publicado por Permanyer. Este es un artículo open access bajo la licencia CC BY-NC-ND (http://creativecommons.org/licenses/by-nc-nd/4.0/) 
of the present study is to analyze the feasibility and the short and medium-term outcomes of the transcatheter closure of paravalvular leaks. Methods: Single-centre registry of consecutive patients with post-surgical paravalvular leaks, that underwent transcatheter closure, between January 2006 and December 2016. Efficacy and safety results were analyzed during the procedure and at 6-month follow-up. Results: 21 paravalvular leaks (15 mitral, 5 aortic and 1 tricuspid) were closure during 20 procedures. In the initial echocardiography $91 \%$ of the leaks were severe. The most used device was the Amplatzer Vascular Plug $11{ }^{\boxplus}$ in 10 procedures (50\%). The 3D transesophageal echocardiography was used in $70 \%$ of cases. The device was successfully implanted in $95 \%$ of cases, a regurgitation reduction $\geq 1$ grade was achieved in $95 \%$ of the cases, and the clinical success was $79 \%$. Six month survival was 100\%; however, 3 cases required valvular surgery (15\%). Conclusions: Transcatheter closure of paravalvular leaks is a feasible and safe procedure with high rates of technical, echocardiographic and clinical success in the short and medium term. It is an adequate therapeutic alternative, mainly in high surgical risk patients and multiples comorbidities.

Key words: Paravalvular leak. Transcatheter closure. Results.

\section{Introducción}

La fuga paravalvular es una complicación relativamente frecuente e importante, que ocurre en 5 a $17 \%$ de las prótesis valvulares quirúrgicas ${ }^{1-3}$, como consecuencia de una falla de la sutura quirúrgica favorecida por la presencia de calcio, infección, friabilidad del tejido o la forma no circular del anillo4,5.

Aunque la mayor parte de las fugas paravalvulares (FPV) es pequeña y asintomática, un 2 a 5\% $\%^{4,6,7}$ tiene relevancia clínica y puede ocasionar insuficiencia cardiaca y anemia hemolítica y está asociada a malos resultados clínicos ${ }^{1,3,8,9}$.

La reintervención quirúrgica, para el cierre del defecto o la sustitución protésica, ha sido el tratamiento clásico. Ha mostrado menor mortalidad en comparación con el tratamiento conservador de las FPV 8,10 , pero se acompaña de una elevada morbimortalidad perioperatoria, así como una alta tasa de fugas residuales o recurrentes, sobre todo en presencia de múltiples operaciones previas y comorbilidades. Además, en la práctica clínica algunos pacientes no pueden intervenirse debido a su riesgo quirúrgico aumentado, lo que implica un mal pronóstico $0^{4,11-15}$.

El cierre transcatéter es una alternativa terapéútica emergente que ha demostrado reducir la gravedad de la fuga y sus síntomas, con tasas de éxito variables y una tasa baja de complicaciones. La localización de las FPV, su morfología, número, tamaño, trayecto, así como el acceso, la técnica y los dispositivos utilizados se han descrito como factores que pueden influir en los resultados ${ }^{6,14,16-18}$. Las fugas paravalvulares tienen características anatómicas variables, lo que hace muy difícil que un dispositivo se ajuste en todos los casos. La selección específica del dispositivo se basa en el tamaño, la forma y la ubicación de la fuga, con la intención de ocluir por completo el defecto sin interferir con la función valvular. Se han usado múltiples dispositivos no diseñados de manera específica para este cometido, entre ellos los dispositivos del grupo Amplatzer (Abbott), que son los más utilizados en la actualidad: el Amplatzer Vascular Plug III (AVP III) en Europa y el AVP II en EE.UU. ${ }^{19-22}$

El objetivo de este estudio es analizar la factibilidad y los resultados inmediatos y a seis meses del cierre transcatéter de las FPV posquirúrgicas con el uso de dispositivos de oclusión en la institución de los autores.

\section{Métodos}

Se empleó el registro de un centro único de una serie consecutiva de pacientes con FPV de protésicas quirúrgicas, las cuales se cerraron vía transcatéter con dispositivos oclusores en el Instituto Nacional de Cardiología Ignacio Chávez de México, entre enero del 2006 y diciembre del 2016.

Se definió la fuga paravalvular como la presencia de un chorro de regurgitación por ecocardiografía Doppler originado entre el borde del anillo protésico y el tejido nativo circundante. La gravedad de la regurgitación se definió de acuerdo con los parámetros establecidos por la American Society of Echocardiography ${ }^{23-25}$. La técnica de cierre transcatéter, la vía de acceso, el tipo del dispositivo implantado y la utilización o no de la ecocardiografía durante el procedimiento se eligieron a criterio de los operadores. Por lo general, para la elección del tamaño del dispositivo se tuvieron en cuenta los diámetros mayor y menor del defecto determinado mediante ecocardiografía transesofágica bidimensional o tridimensional y se utilizó un dispositivo con un tamaño $\geq 1-2 \mathrm{~mm}$ que los diámetros de referencia.

Para todos los casos, las características basales, los hallazgos del ecocardiograma, los detalles del procedimiento, los resultados hospitalarios y el seguimiento 
se recolectaron del expediente médico electrónico. Todos los pacientes cumplieron un seguimiento clínico mínimo de seis meses, durante el cual se sometieron a un estudio ecocardiográfico control. Se buscaron los resultados del seguimiento respecto al grado de fuga residual, cambio en la clase funcional, complicaciones vasculares, embolización al dispositivo, muerte, infarto de miocardio, necesidad de operación valvular posterior al implante del dispositivo, hemólisis, reintervención, accidente cerebrovascular, endocarditis infecciosa y rehospitalización. Tras culminar el procedimiento se definió el éxito técnico como el implante del dispositivo a nivel de la fuga paravalvular sin interferencia con el funcionamiento normal de la prótesis o necesidad de una intervención urgente, y el éxito del procedimiento cuando, además del éxito técnico, se consiguió una reducción $\geq 1$ grado de la fuga paravalvular. Se valoró la clase funcional basal y a los seis meses de acuerdo con la clasificación de la New York Heart Association. Se consideró éxito clínico en el seguimiento cuando se constató una mejoría clínica $\geq 1$ grado en la clase funcional en los siguientes 180 días luego del procedimiento de cierre. La anemia hemolítica se definió por la documentación clínica de síntomas en conjunción con pruebas de laboratorio de anemia y hemólisis ${ }^{16}$.

\section{Análisis estadísticos}

Las variables cualitativas se presentan como número absoluto y porcentaje, y las variables cuantitativas en forma de media y desviación estándar. El análisis estadístico se llevó a cabo con el programa SPSS versión 21.0 (SPSS Inc., Chicago, Illinois, EE.UU.).

\section{Resultados}

Durante el periodo analizado se intervinieron 21 prótesis valvulares (15 mitrales, 5 aórticas y 1 tricuspídea) durante 20 procedimientos. En un paciente se cerraron dos fugas en diferentes localizaciones (mitral y tricuspídea) en un mismo procedimiento. Un $58 \%$ de los casos correspondió a varones, la edad promedio fue de $45 \pm 18$ años y un $50 \%$ de casos presentaba dos o más esternotomías previas. El tiempo promedio desde la operación valvular hasta el cierre de la FPV fue de 9.4 años. Las características demográficas y clínicas basales se resumen en la Tabla 1.

Las indicaciones más frecuentes del procedimiento fueron la insuficiencia cardiaca o la anemia hemolítica en un $75 \%$ de los casos y la mayor parte de éstos (70\%) se presentó en las clases funcionales II a IV de
Tabla 1. Características clínicas basales de los pacientes

\begin{tabular}{|c|c|}
\hline Características demográficas y clínicas & $\mathrm{n}=20$ \\
\hline Edad promedio & $45( \pm 18)$ \\
\hline Varones & $11(58 \%)$ \\
\hline $\begin{array}{l}\text { Localización protésica de las FPV } \\
\text { - Mitral } \\
\text { - Aórtica } \\
\text { - Tricuspídea } \\
\text { Prótesis mecánica }\end{array}$ & $\begin{array}{c}15(71 \%) \\
5(24 \%) \\
1(5 \%) \\
17(81 \%)\end{array}$ \\
\hline $\begin{array}{l}\text { Antecedentes } \\
\text { - Fibrilación auricular } \\
\text { - Cardiopatía isquémica } \\
\text { - Hipertensión arterial } \\
\text { - Endocarditis } \\
\text { - Diabetes mellitus } \\
\text { - ECV previo } \\
\text { - ERC (TFG < } 60 \mathrm{ml} / \mathrm{min} \text { ) } \\
\text { - EPOC } \\
\text { Hipertensión pulmonar (PSAP } \geq 40 \mathrm{mmHg} \text { ) } \\
\text { Tiempo promedio desde la operación } \\
\text { valvular hasta la intervención } \\
\text { Portador de una válvula protésica } \\
\geq 2 \text { esternotomías previas } \\
\text { Clase funcional basal, NYHA } \geq \text { II }\end{array}$ & $\begin{array}{l}7(35 \%) \\
4(20 \%) \\
4(20 \%) \\
2(10 \%) \\
1(5 \%) \\
1(5 \%) \\
0(0 \%) \\
0(0 \%) \\
12(60 \%) \\
9.4 \text { años } \\
16(80 \%) \\
10(50 \%) \\
14(70 \%)\end{array}$ \\
\hline $\begin{array}{l}\text { Indicación del procedimiento } \\
\text { - Insuficiencia cardiaca } \\
\text { - Anemia hemolítica } \\
\text { - IC + AH } \\
\text { - Hallazgo ecocardiográfico }\end{array}$ & $\begin{array}{c}10(50 \%) \\
2(10 \%) \\
3(15 \%) \\
5(25 \%)\end{array}$ \\
\hline
\end{tabular}

FPV: fuga paravalvular; ECV: enfermedad cerebrovascular; ERC: enfermedad renal crónica, determinada por una tasa de depuración de creatinina calculada $\leq 60 \mathrm{ml} / \mathrm{min}$; EPOC: enfermedad pulmonar obstructiva crónica; PSAP: presión sistólica de la arteria pulmonar, calculada por ecocardiografía; NYHA: Clasificación de la New York Heart Association; IC: insuficiencia cardiaca; AH: anemia hemolítica.

la NYHA. En 10 pacientes (50\%) se describieron múltiples fugas (en el $91 \%$ de los casos éstas fueron graves) y la fracción de eyección del ventrículo izquierdo fue en promedio de $54 \pm 17 \%$ (Tabla 2).

El acceso fue femoral en todos los individuos; en cuanto a las FPV aórticas, todos los casos se practicaron mediante abordaje retrógrado, y en el caso de las FPV mitrales un $87 \%$ de casos se realizó un $87 \%$ de los casos se realizó un abordaje anterógrado a través de una punción transeptal, mientras que el $13 \%$ restante se abordó de forma retrógrada desde el ventrículo izquierdo hasta la aurícula izquierda (Tabla 3).

Se logró implantar el dispositivo con éxito (técnico) en el $95 \%$ de las veces, sólo en un caso ocurrió una embolización del dispositivo y se consiguió la reducción $\geq 1$ grado de regurgitación (éxito del procedimiento) en el $95 \%$ de los casos. Se implantaron 25 dispositivos; el utilizado más a menudo fue el Amplatzer Vascular 
Tabla 2. Características ecocardiográficas basales, previas

\begin{tabular}{|l|c|}
\hline $\begin{array}{l}\text { Número de fugas presentes por válvula } \\
\text { - Una } \\
\text { - Múltiples ( } \geq 2 \text { ) }\end{array}$ & $10(50 \%)$ \\
\hline $\begin{array}{l}\text { Localización de la fuga paravalvular mitral }(\mathrm{n}=17 \\
\text { fugas) } \\
\text { - Posterior } \\
\text { - Anterior } \\
\text { - Lateral }\end{array}$ & $9(53 \%)$ \\
\hline $\begin{array}{l}\text { Localización de la fuga paravalvular } \\
\text { aórtica (n }=7 \text { fugas) } \\
\text { - Anterior } \\
\text { - Posterior } \\
\text { - Lateral }\end{array}$ & $5(29 \%)$ \\
\hline $\begin{array}{l}\text { Localización de la fuga paravalvular } \\
\text { tricuspídea (n = fuga) } \\
\text { - Septal }\end{array}$ & $3(18 \%)$ \\
\hline $\begin{array}{l}\text { Grado de fuga paravalvular antes del } \\
\text { procedimiento } \\
\text { - Grave } \\
\text { - Moderada }\end{array}$ & $2(43 \%)$ \\
\hline \begin{tabular}{l} 
FEVI (\%) promedio \\
\hline
\end{tabular} & $2(28 \%)$ \\
\hline
\end{tabular}

FEVI: fracción de expulsión del ventrículo izquierdo.

Plug III ${ }^{\circledR}$ en 13 de las fugas cerradas (52\%), seguido por el Amplatzer Vascular Plug ${ }^{\circledR}{ }^{\circledR}$ en cuatro fugas (16\%) y el Amplatzer de Persistencia de Conducto Arterioso ${ }^{\circledR}$ en otras cuatro (16\%); de manera adicional se usaron Amplatzers de Comunicación Interauricular ${ }^{\circledR}$ en dos fugas (8\%) y Amplatzers de Comunicación Interventricular $^{\circledR}$ en dos más (8\%) (Figuras 1, 2 y 3). En cinco procedimientos se utilizaron dos oclusores debido al tamaño, la forma de la fuga objetivo o múltiples fugas en la misma válvula. Se empleó ecocardiograma transesofágico en un $90 \%$ de los procedimientos $(70 \%$ con uso de técnica tridimensional), ecocardiograma intracardiaco en un caso (5\%) y se guio el procedimiento sólo por fluoroscopia en un caso (5\%) (Tabla 3).

El grado de fuga residual fue nulo o leve en $80 \%$ de los casos y a seis meses se obtuvo un éxito clínico de $79 \%$. No se presentaron complicaciones vasculares relacionadas con el procedimiento ni episodios cerebrales vasculares en el seguimiento. A seis meses, la supervivencia fue del 100\%; sin embargo, tres casos requirieron operación valvular (15\%), un caso de forma urgente por la embolización del dispositivo y los otros dos por la aparición de anemia hemolítica. No hubo ningún caso de interferencia con el funcionamiento normal de la prótesis (Tabla 4).
Tabla 3. Características del procedimiento

\begin{tabular}{|c|c|}
\hline \multicolumn{2}{|l|}{ Técnica de cierre } \\
\hline $\begin{array}{l}\text { Fugas mitrales } \\
\text { - Anterógrada } \\
\text { - Retrógrada }\end{array}$ & $\begin{array}{c}n=15 \\
13(87 \%) \\
2(13 \%)\end{array}$ \\
\hline $\begin{array}{l}\text { Fugas aórticas } \\
\text { - Retrógrada }\end{array}$ & $\begin{array}{c}n=5 \\
5(100 \%)\end{array}$ \\
\hline $\begin{array}{l}\text { Fuga tricuspídea } \\
\text { - Anterógrada } \\
\text { - Éxito técnico }\end{array}$ & $\begin{array}{c}n=1 \\
1(100 \%) \\
19(95 \%)\end{array}$ \\
\hline $\begin{array}{l}\text { Tipo de dispositivo utilizado ( } \mathrm{n}=25 \\
\text { dispositivos) } \\
\text { - Amplatzer Vascular Plug III } \\
\text { - Amplatzer Vascular Plug II } \\
\text { - Amplatzer CIA } \\
\text { - Amplatzer PCA } \\
\text { - Amplatzer CIV }\end{array}$ & $\begin{array}{l}13(52 \%) \\
4(16 \%) \\
2(8 \%) \\
4(16 \%) \\
2(8 \%)\end{array}$ \\
\hline $\begin{array}{l}\text { Número de dispositivos utilizados en } \\
\text { cada FPV } \\
-1 \text { oclusor } \\
-2 \text { oclusores }\end{array}$ & $\begin{array}{c}15(75 \%) \\
5(25 \%)\end{array}$ \\
\hline $\begin{array}{l}\text { Uso de la ecocardiografía durante el } \\
\text { procedimiento } \\
\text { - ETE } 3 D \\
\text { - ETE } \\
\text { - EIC } \\
\text { - IC + ETE } \\
\text { - No, sólo fluoroscopia }\end{array}$ & $\begin{array}{c}14(70 \%) \\
2(10 \%) \\
1(5 \%) \\
2(10 \%) \\
1(5 \%)\end{array}$ \\
\hline $\begin{array}{l}\text { Fuga residual tras el procedimiento } \\
\text { - Ausente/leve } \\
\text { - Moderada/grave } \\
\text { Tiempo promedio de fluoroscopia } \\
\text { Anestesia general } \\
\text { Tiempo promedio de seguimiento }\end{array}$ & $\begin{array}{c}16(80 \%) \\
4(20 \%) \\
55 \text { minutos } \\
19(95 \%) \\
26 \text { meses }\end{array}$ \\
\hline
\end{tabular}

CIA: comunicación interauricular; PCA: persistencia del conducto arterioso; CIV: comunicación interventricular; ETE 3D: ecocardiograma transesofágico tridimensional; ETE: ecocardiograma transesofágico; EIC: ecocardiograma intracardiaco; FPV: fuga paravalvular.

\section{Discusión}

La reoperación quirúrgica es el tratamiento regular para el tratamiento de las FPV; sin embargo, múltiples series registran una mortalidad a 30 días de 6 a 22\%. Taramasso, et al. informaron un éxito del procedimiento quirúrgico del $98 \%$ en 122 pacientes, pero con una mortalidad a 30 días del $11 \%$, todos los casos en relación con causas cardiacas, lo cual sugiere que la intervención es de alto riesgo. Estos resultados apoyan la necesidad de una alternativa terapeútica válida para la operación convencional, sobre todo en pacientes con múltiples intervenciones cardiacas previas y factores de riesgo para mayor mortalidad (comorbilidades adjuntas, insuficiencia renal crónica, FPV mitral) ${ }^{26}$. 


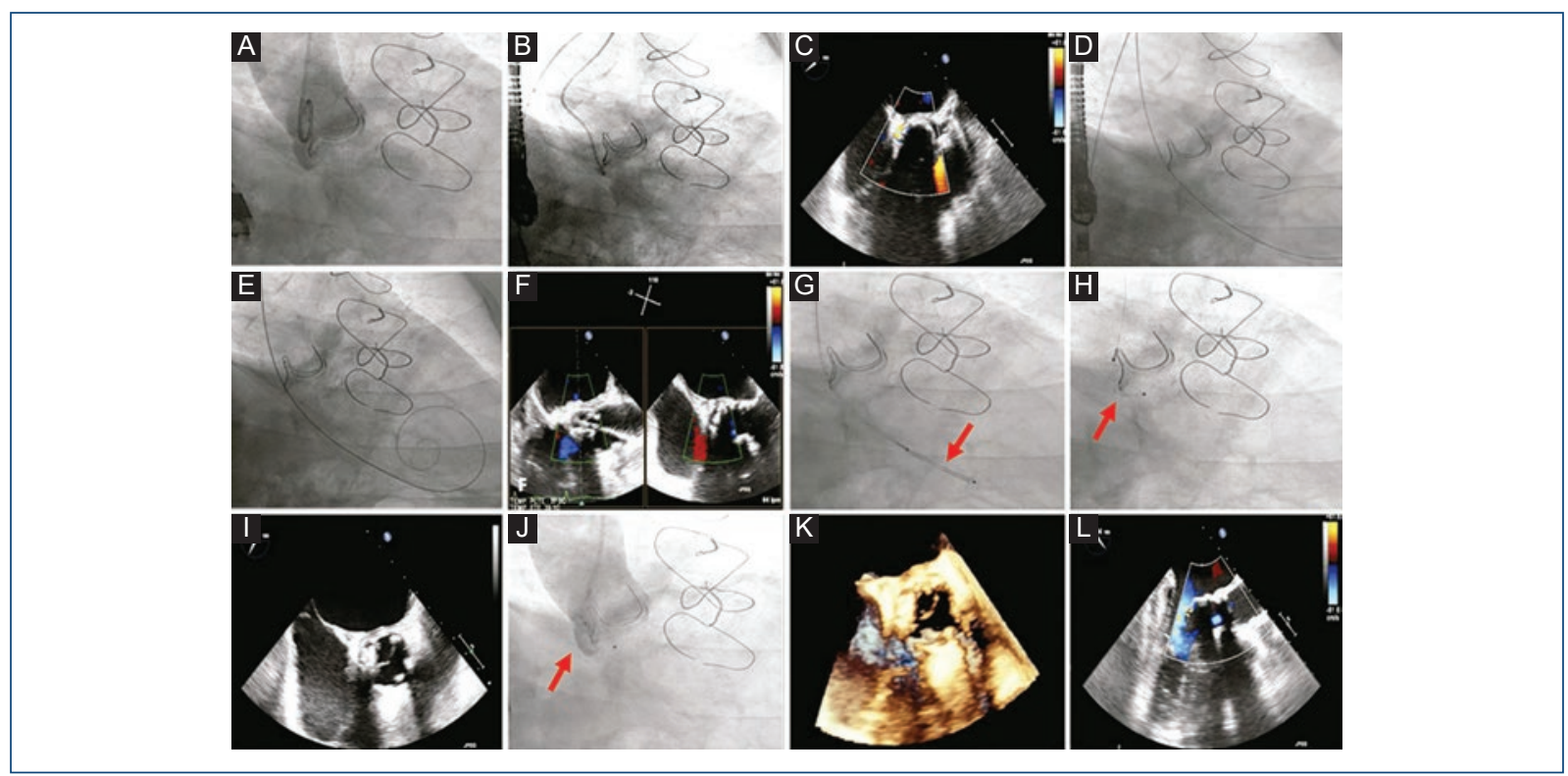

Figura 1. Cierre transcatéter de una fuga paravalvular aórtica. Amplatzer Vascular Plug II 8 mm (fechas rojas). A: aortograma, regurgitación paravalvular aórtica grave. B: canulación selectiva de la fuga. C: ecocardiograma transesofágico (ETE), verificación de la posición y gravedad de la fuga. D: cruce retrógrado a través del defecto. E: intercambio por una guía rígida. F: verificación ecocardiográfica de la posición de la guía. G: avance del catéter y liberación del disco de retención inicial del oclusor. H: liberación del disco de retención final del oclusor. I: evaluación de la posición del dispositivo y del funcionamiento valvular. J: aortograma final, regurgitación residual leve. K-L: resultado final mediante ETE tridimensional y bidimensional.

Tabla 4. Resultados clínicos a seis meses

\begin{tabular}{|l|c|}
\hline Clase funcional final NYHA I & $17(85 \%)$ \\
\hline Complicación vascular del acceso & 0 \\
\hline Operación valvular & $3(15 \%)$ \\
\hline Embolización del dispositivo & $1(5 \%)$ \\
\hline Anemia hemolítica nueva & $2(10 \%)$ \\
\hline Enfermedad cerebrovascular & 0 \\
\hline Muerte por todas las causas & 0 \\
\hline
\end{tabular}

NYHA: Clasificación de la New York Heart Association.

En la serie de los autores se consideró para elegir el cierre transcatéter, además del riesgo quirúgico, el número de esternotomías previas $(50 \%$ presentaba más de una) y la edad del paciente (45 años en promedio), con el fin de evitar una reintervención y una exposición a un mayor número de reesternotomías futuras, ya que existe un incremento progresivo de la mortalidad quirúrgica con el número de reoperaciones.

El éxito técnico en la serie fue alto, similar al de un metaanálisis de 12 estudios no aleatorizados en el que se publicó una cifra de $76.5 \%{ }^{17}$. Esto sugiere que, a pesar de ser un procedimiento técnicamente complejo, la experiencia de los centros, el trabajo en equipo y el uso de técnicas de imagen apropiadas permiten obtener buenos resultados en la mayoría de los casos.

El éxito del procedimiento también fue elevado, semejante al de las series publicadas de Ruiz, et al. ${ }^{6}(86 \%)$ y Sorajja et al. ${ }^{18}(89 \%)$, pero a diferencia de la serie de los autores en aquéllas no se utilizó el dispositivo AVP III, principal oclusor empleado en ésta, lo cual podría tener relación con la elevada tasa de éxito del procedimiento encontrada, dado que su morfología ovalada podría adaptarse mejor a la anatomía (casi siempre semilunar) del defecto, viene premontado, tiene mejor perfil de cruce y se puede introducir por vainas de menor calibre. En cinco casos (25\%) se requirió el uso de dos oclusores, lo cual muestra la ausencia de un dispositivo ideal para todos los casos y que, en defectos grandes e irregulares, se pueden amoldar múltiples dispositivos pequeños mejor al espacio paravalvular y evitar la disfunción protésica.

Un 95\% de los casos de los autores tuvo guía ecocardiográfica, ya que ésta juega un papel crucial durante el procedimiento y dirige al operador durante las diferentes fases de la intervención, como la elección del lugar adecuado para la punción transeptal, el cruce adecuado de 


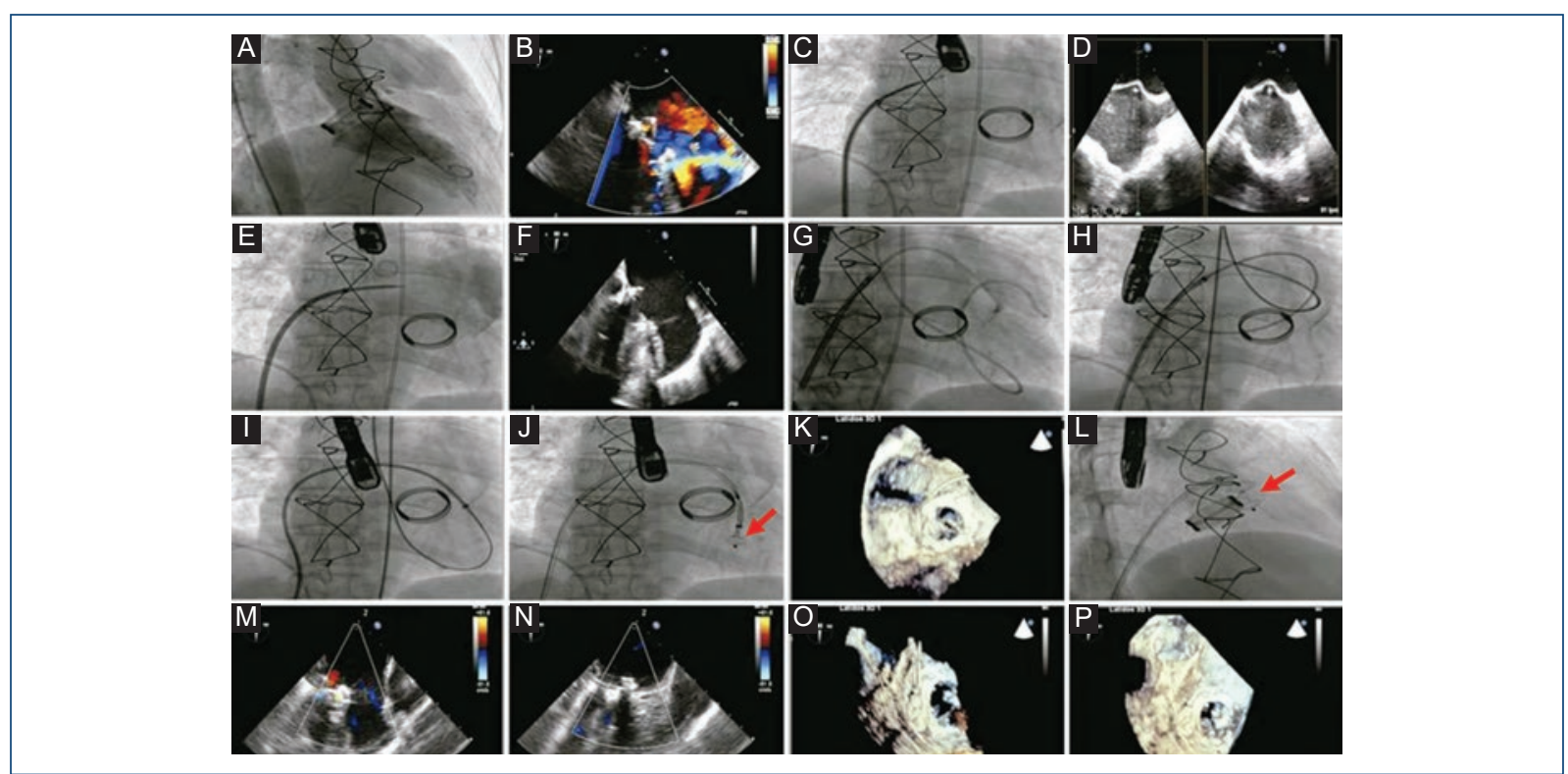

Figura 2. Cierre transcatéter de una fuga paravalvular mitral. Amplatzer Vascular Plug II 12/25 mm (fechas rojas). A: ventriculografía izquierda, regurgitación paravalvular mitral grave. B: Ecocardiograma transesofágico (ETE), verificación de la posición y gravedad de la fuga. C: Punción transeptal. D: ETE que guía el nivel de la punción transeptal. E: Angiografía a nivel de la aurícula izquierda. F: Cruce retrógrado de la guía desde el ventrículo izquierdo hasta la aurícula izquierda a través del defecto, verificado mediante ecocardiografía. G-H: Lazo a nivel de la aurícula izquierda y formación de un asa arteriovenosa. I: Avance anterógrado del sistema de liberación a través del defecto. J: Liberación del disco de retención inicial del oclusor. K: Evaluación de la posición del dispositivo y del funcionamiento valvular. L: Liberación del disco de retención final del oclusor. M-P: Resultado final mediante ETE bidimensional y tridimensional.

la guía a través del defecto, la elección del dispositivo y la evaluación inmediata del resultado siendo; la gravedad de la fuga y su tamaño mejor estimadas mediante ecocardiografía transesofágica tridimensional. En los últimos años se ha descrito que la nueva tecnología de fusión de imágenes de ecocardiografía tridimensional con fluoroscopia puede ayudar a facilitar el éxito del procedimiento y evaluar mejor el resultado 27,28 .

En la serie más recientemente publicada, el éxito clínico a 30 días fue del $72 \%{ }^{29}$, semejante al de la serie de los autores, en la cual $79 \%$ de los casos mejoró sus síntomas a seis meses. Existe escasa evidencia que compare el cierre transcatéter respecto de la intervención quirúrgica. Un estudio comparativo no aleatorizado, publicado por Wells, et al., notificó resultados clínicos a un año equivalentes entre el cierre transcatéter y la intervención quirúrgica, con significativamente menor estancia postoperatoria, estancia en la unidad de cuidados intensivos, readmisión a los 30 días, sangrado y morbilidad perioperatoria. Otro estudio comparativo de Angulo-Llanos, et al. señaló que la mortalidad intrahospitalaria fue significativamente mayor en el grupo quirúrgico $(30.6 \%$ vs. $9.8 \%)$ y la mejoría clínica significativamente mayor en el grupo transcatéter ${ }^{13,29}$. Taramasso, et al. encontraron que el cierre transcatéter vía transapical en pacientes de alto riesgo tuvo una mortalidad hospitalaria menor en comparación con el cierre quirúrgico y la sobrevida general a 12 años fue de $39.8 \pm 7 \%$ (siendo significativamente menor en pacientes con más de una reoperación $\left.{ }^{15}\right)$.

La mayor parte de los pacientes (80\%) de los autores tuvo una fuga residual nula o leve, lo cual parece clave para obtener un buen resultado, puesto que se ha descrito una relación significativa con la tasa de episodios adversos cardiovasculares, la clase funcional final y una mejor sobrevida a largo plazo ${ }^{30}$.

El procedimiento representa muchos retos durante su desarrollo y no está exento de complicaciones, por lo cual es fundamental una vigilancia constante. La tasa de complicaciones publicada es baja y las más frecuentes son las vasculares, la operación urgente por embolización del dispositivo, la interferencia con la prótesis, el ictus perioperatorio y las hemorragias; a pesar de todo esto, la muerte relacionada con el procedimiento es poco frecuente ${ }^{6,19,30-32}$, lo cual sugiere que éste es un procedimiento seguro, semejante a los hallazgos de 


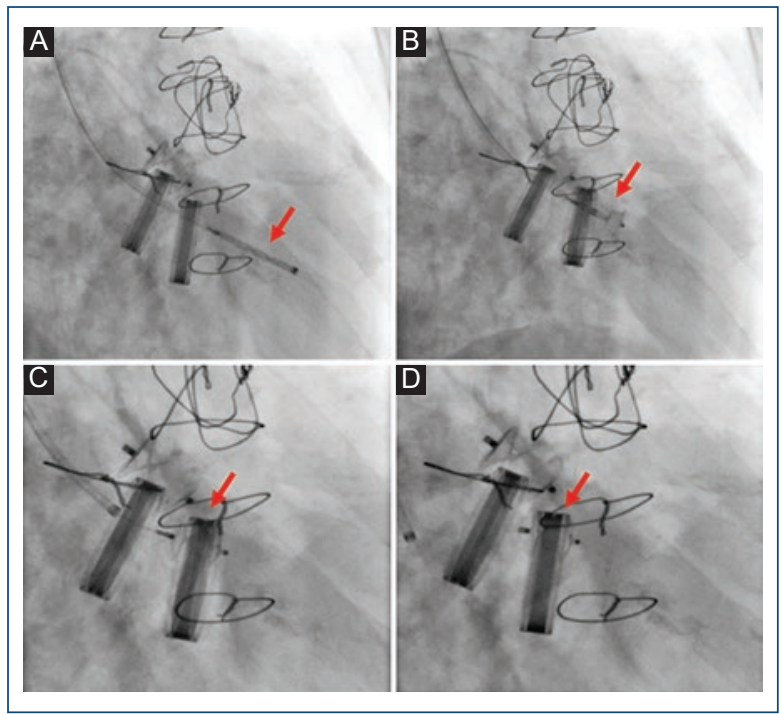

Figura 3. Cierre transcatéter de fuga paravalvular tricuspídea. Amplatzer Vascular Plug II 8/4 mm (fechas rojas). A: cruce anterógrado del defecto desde el acceso yugular y avance del sistema de liberación. B: liberación del disco de retención inicial del oclusor. C: liberación del disco de retención final del oclusor. D: resultado final mediante fluoroscopia.

los autores en los cuales la principal complicación aguda fue la embolización de un dispositivo (5\%). Por otro lado, se presentó durante el seguimiento una nueva anemia hemolítica en dos pacientes (10\%), en quienes se usaron oclusores Amplatzer de tabique auricular y ventricular, lo cual obligó a una nueva intervención valvular, algo significativamente mayor a lo informado con anterioridad de sólo un $1.6 \%$. Por consiguiente, el desarrollo de dispositivos dedicados es necesario para sobrepasar este importante problema. Asimismo, se ha descrito la insuficiencia cardiaca terminal como la causa más frecuente de muerte de estos individuos en el seguimiento ${ }^{33,34}$; esto señala que el cierre transcatéter de las fugas se suele realizar en un estadio avanzado de la enfermedad valvular o en presencia de múltiples comorbilidades.

Por último, los resultados apoyan el cierre transcatéter de las FPV como una alternativa menos invasiva, efectiva y segura, en manos de un equipo multidisciplinario y tras una detallada planeación. Hay que mencionar como limitación que este trabajo se basó en un registro único, de una serie pequeña de pacientes con un seguimiento a mediano plazo.

\section{Conclusiones}

El cierre transcatéter de fugas paravalvulares es un procedimiento factible, seguro y con elevadas tasas de éxito técnico, ecocardiográfico y clínico a corto y mediano plazo. Es una alternativa terapéutica adecuada, en particular en pacientes de alto riesgo quirúrgico o múltiples comorbilidades asociadas. Aún son necesarios estudios comparativos a largo plazo para definir al cierre transcatéter como la primera estrategia de tratamiento de las fugas paravalvulares.

\section{Conflicto de intereses}

Los autores declaran no tener ningún conflicto de intereses.

\section{Financiamiento}

No se recibió patrocinio de ningún tipo para llevar a cabo este artículo.

\section{Responsabilidades éticas}

Protección de personas y animales. Los autores declaran que para esta investigación no se han realizado experimentos en seres humanos ni en animales.

Confidencialidad de los datos. Los autores declaran que han seguido los protocolos de su centro de trabajo sobre la publicación de datos de pacientes.

\section{Derecho a la privacidad y consentimiento infor-} mado. Los autores declaran que en este artículo no aparecen datos de pacientes.

\section{Bibliografía}

1. Kodali SK, Williams MR, Smith CR, Svensson LG, Webb JG, Makkar RR et al. Two-year outcomes after transcatheter or surgical aortic-valve replacement. N Engl J Med. 2012; 366:1686-95.

2. Rihal CS, Sorajia $P$, Booker JD, Hagler DJ, Cabalka AK. Principles of percutaneous paravalvular leak closure. J Am Coll Cardiol Intv. 2012; 5:121-30.

3. Leon MB, Smith CR, Mack MJ, Makkar RR, Svensson LG, Kodali SK, et al. Transcatheter or surgical aortic-valve replacement intermediate-risk patients. N Engl J Med. 2016;374:1609-20.

4. Miller DL, Morris JJ, Schaff HV, Mullany CJ, Nishimura RA, Orszulak TA. Reoperation for aortic valve periprosthetic leakage: identification of patients at risk and results of operation. J Heart Valve Dis. 1995;4:160-5.

5. Rallidis LS, Moyssakis IE, Ikonomidis I, Nihoyannopoulos P. Natural history of early aortic paraprosthetic regurgitation: a five-year follow-up. Am Heart J. 1999;138:351-7.

6. Ruiz CE, Jelnin V, Kronzon I, Dudiy Y, Del Valle-Fernández R. Clinical outcomes in patients undergoing percutaneous closure of periprosthetic paravalvular leaks. J Am Coll Cardiol. 2011;58:2210-7.

7. Jindani A, Neville EM, Venn G, Williams BT. Paraprosthetic leak: a complication of cardiac valve replacement. J Cardiovasc Surg (Torino). 1991;32:503-8.

8. Genoni M, Franzen D, Vogt P, Seifert B, Jenni R, Künzli A, et al. Paravalvular leakage after mitral valve replacement: improved long-term survival with aggressive surgery? Eur J Cardio-Thorac Surg. 2000;17:14-9.

9. Hein R, Wunderlich N, Robertson G, Wilson N, Sievert H. Catheter closure of paravalvular leak. Eurolntervention. 2006; 2:318-25.

10. Cho IJ, Hong GR, Lee S, Byung-Chul C, Ha JW, Chung N. Predictors of prognosis in patients with mild to moderate paravalvular leakage after mitral valve replacement. J Card Surg. 2014;29:149-154.

11. Akins CW, Bitondo JM, Hilgenberg AD, Vlahakes GJ, Madsen JC, MacGillivray TE. Early and late results of the surgical correction of cardiac prosthetic paravalvular leaks. J Heart Valve Dis. 2005;14:792-9. 
12. Taramasso $M$, Maisano $F$, Denti $P$, et al. Surgical treatment of paravalvular leak: long term results in a single-center experience (up to 14 years). J Thorac Cardiovasc Surg. 2015;149:1270-5.

13. Angulo-Llanos $R$, Sarnago-Cebada F, Rivera AR, Elízaga Corrales J, Cuerpo G, Solis J, et al. Two-year follow up after surgical versus percutaneous paravalvular leak closure: a nonrandomized analysis. Catheter Cardiovasc Interv. 2016; 88:626-34.

14. Said SM. Paravalvular leak, the Achilles' heel of valve replacement: Will transcatheter techniques be the answer? J Thorac Cardiovasc Surg. 2015;149:1276-7.

15. Taramasso M, Maisano F, Latib A, Denti P, Guidotti A, Sticchi A, et al. Conventional surgery and transcatheter closure via surgical transapical approach for paravalvular leak repair in high-risk patients: results from a singlecentre experience. Eur Heart J Cardiovasc Imaging. 2014;15:1161-7.

16. Millan X, Skaf S, Joseph L, Ruiz C, García E, Smolka G, et al. Transcatheter reduction of paravalvular leaks: a systematic review and meta-analysis. Can J Cardiol. 2015;31:260-9.

17. Okuyama K, Jilaihawi $\mathrm{H}$, Kashif M, Soni V, Matsamuto T, Yeow WL, et al. Percutaneous paravalvular leak closure for balloon-expandable transcatheter aortic valve replacement: a comparison with surgical aortic valve replacement paravalvular leak closure. J Invasive Cardiol. 2015; 27:284-90.

18. Sorajja P, Cabalka AK, Hagler DJ, Rihal CS. Percutaneous repair of paravalvular prosthetic regurgitation: acute and 30-day outcomes in 115 patients. Circ Cardiovasc Interv. 2011:4:314-21.

19. Ozkan M, Gursoy OM, Astarcioglu MA, Wunderlich N, Sievert H. Percutaneous closure of paravalvular mitral regurgitation with Vascular Plug III under the guidance of real-time three-dimensional transesophageal echocardiography. Turk Kardiyol Dern Ars. 2012;40:632-41.

20. Smolka G, Pysz P, Jasinski M. Two-stage percutaneous closure of paravalvular leak in a patient with stentless aortic bioprosthesis. Catheter Cardiovasc Interv. 2013;82:E119-23.

21. Nietlispach F, Johnson M, Moss RR, Wireline N, Gurvitch R, Tay EL, et al. Transcatheter closure of paravalvular defects using a purpose-specific occluder. JACC Cardiovasc Interv. 2010;3:759-65.

22. Moreno R, Sánchez Recalde A, López Fernández T, Moreno-Gómez I, Mesa JM, López Sendon JL. Cierre percutáneo de fugas perivalvulares mitrales en pacientes con prótesis metálica aórtica. Rev Esp Cardiol. 2012;65:768-9.

23. Lancellotti P, Tribouilloy C, Hagendorff A, Popescu BA, Edvardsen T, Pierard LA, et al. Recommendations for the echocardiographic assessment of native valvular regurgitation: an executive summary from the European Association of Cardiovascular Imaging. Eur Heart J Cardiovasc Imaging. 2013;14:611-44.
24. Zamorano J, Goncalves A, Lancellotti P, Andersen KA, González-Gómez A Monaghan $M$, et al. The use of imaging in new transcatheter interventions: an EACVI review paper. Eur Heart J Cardiovasc Imaging. 2016;17:835-835af.

25. Zoghbi WA, Chambers JB, Dumesnil JG, Foster E Gottdiener JS, Grayburn $\mathrm{PA}$, et al. Recommendations for evaluation of prosthetic valves with echocardiography and Doppler ultrasound: a report from the American Society of Echocardiography's Guidelines and Standards Committee and the Task Force on Prosthetic Valves, developed in conjunction with the American College of Cardiology Cardiovascular Imaging Committee, Cardiac Imaging Committee of the American Heart Association, the European Association of Echocardiography, a registered branch of the European Society of Cardiology, the Japanese Society of Echocardiography and the Canadian Society of Echocardiography, endorsed by the American College of Cardiology Foundation, American Heart Association, European Association of Echocardiography, a registered branch of the European Society of Cardiology, the Japanese Society of Echocardiography, and Canadian Society of Echocardiography. J Am Soc Echocardiogr. 2009;22:975-1014.

26. Taramasso M, Maisano F, Denti P, Guidotti A, Sticchi A, Pozzoli A, et al. Surgical treatment of paravalvular leak: long-term results in a single-center experience (up to 14 years). J Thorac Cardiovasc Surg. 2015;149:1270-5.

27. Anwar AM, Nosir YF, Zainal-Abidin SK, Ajam A, Chamsi-Pasha H. Real-time three-dimensional transthoracic echocardiography in daily practice: initial experience. Cardiovasc Ultrasound. 2012;10:14.

28. Mele D, Agricola E, Dal Monte A, Ancona MB, Ferrari R. Perivalvular leak assessment and closure: role of real-time three-dimensional transesophageal echocardiography. G Ital Cardiol (Rome). 2012;13:38-46.

29. Wells JA $4^{\text {th }}$, Condado JF, Kamioka N, Dong A, Ritter A, Lerakis S, et al. Outcomes after paravalvular leak closure: transcatheter versus surgical approaches. JACC Cardiovasc Interv. 2017;10(5):500-507

30. Shapira Y, Hirsch R, Kornowski R, Hasdai D, Assali A, Vaturi M, et al. Percutaneous closure of perivalvular leaks with amplatzer occluders: feasibility, safety, and shortterm results. J Heart Valve Dis. 2007;16:305-13.

31. Kim MS, Casserly IP, García JA, Klein AJ, Salcedo EE, Carrol JD, et al. Percutaneous transcatheter closure of prosthetic mitral paravalvular leaks: are we there yet? JACC Cardiovasc Interv. 2009;2:81-90.

32. Pate GE, Al Zubaidi A, Chandavimol M, Thompson CR, Munt BI, Webb JG. Percutaneous closure of prosthetic paravalvular leaks: case series and review. Catheter Cardiovasc Interv. 2006;68:528-33.

33. Hein R, Wunderlich N, Robertson G, Wilson N, Sievert H. Catheter closure of paravalvular leak. Euro Intervention. 2006;2:318-25.

34. Sorajja P, Cabalka AK, Hagler DJ, Rihal CS. Long-term follow-up of percutaneous repair of paravalvular prosthetic regurgitation. J Am Coll Cardiol. 2011;58:2218-24. 